\title{
BFGS Method for Linear Programming
}

\author{
A. Amir and A. Yassine \\ 1. Laboratory of Pure and Applied Mathematics, University of Mostaganem (Algeria) and associate member in Laboratory of \\ Applied Mathematics, University of Lehavre, France. \\ 2. Laboratory of Applied Mathematics, University of Lehavre, France.
}

Received: August 20, 2015 / Accepted: September 17, 2015 / Published: December 25, 2015.

\begin{abstract}
In this paper we propose an algorithm based on the BFGS Quasi-Newton method to solve a linear program. The choice of this method is justified by its theoretical efficiency, the ease to determine a descent direction and its fast convergence towards an optimal solution. Our proposed method is compared with Newton's method for linear program named lpnew, widely used as an optimization algorithm for classification problems.
\end{abstract}

Key Words: Linear programming, BFGS method, least norm solution, exterior penalty.

\section{Introduction}

It may seem strange to solve a linear program with nonlinear optimization methods. Indeed, a gradient type algorithm can lead to a numerical explosion, or at best a bifurcation regime. However, and for example, the interior points methods [14], with their ease of implementation and their polynomial complexity, make nonlinear approaches so interesting for linear programming.

Another technique using nonlinear approach is based on the Tikhonov regularization [15] and the fact that the exterior penalty formulation of a linear program provides an exact least 2-norm solution to the dual of the linear program for finite values of the penalty parameter [9]. This solution is used to generate an accurate primal solution. If $m$ (constraints number) $>>n$ (variables number), a fast globally-convergent and finitely terminating Newton method named lpnewt was proposed by the author in [9] to solve the primal linear program. The algorithm, in question, has been successfully used

Corresponding author: A. Amir, Laboratory of Pure and Applied Mathematics, University of Mostaganem, Algeria and associate member in Laboratory of Applied Mathematics, University of Lehavre, France. amirabdessamad@yahoo.fr to solve several problems in Datamining and Machine Learning ([3], [4]).

However, it is to note that when $m$ and $n$ are of the same order, this method gives a solutions of poor quality. Our objective in this paper, is to develop in this situation an algorithm with the same quality as lpnewt. In fact, since the gradient of the objective function of the penalized linear program is not differentiable, the author in [9] used a generalized modified Newton method. We propose here, to use a quasi-Newton method of type BFGS (Broyden-Fletcher-Goldfarb-Shanno), wich has never been used in this context, instead of the generalized Newton method.The BFGS quasi-Newton method [13] and its variant are widely regarded as the workhorses of smooth nonlinear optimization due to their combination of computational efficiency and good asymptotic convergence. Byrd and Nocedal [1] showed that, if the objective function is convex and twice continuously differentiable with additional assumptions, we have the assurance that the BFGS method combines global convergence with a rate of superlinear convergence. Since then, several studies have shown that we can have a global convergence with weaker assumptions, and some researchers have 
spoken even about the BFGS method for nonsmooth optimization problems ([16], [7]).

The paper is organized as follows: In the second section, we give the theoretical background showing that the least 2-norm formulation of a linear program, as a strongly convex quadratic program, gives an exact least 2-norm solution for finite parameter values ([11], [5]). In section 3, we supply the theoretical results of our method as well as its theorem of convergence. Comparative numerical simulations with the lpnewt method (proposed in [9]) on sparse synthetically-generated linear programs are given to show the superiority and the robustness of our approach. A general conclusion on this work is supplied in the last section.

\section{Theoretical Backgroud}

We consider the primal linear programming problem

$$
\left\{\begin{array}{l}
\min c^{\top} x \\
A x \leq b \text { and } x \in \mathbb{R}^{n}
\end{array}\right.
$$

and its dual

$$
\left\{\begin{array}{l}
-\min b^{\top} v \\
A^{\top} v+c=0 \text { and } v \in \mathbb{R}_{+}^{m}
\end{array}\right.
$$

where $A \in \mathbb{R}^{(m, n)}, \quad c \in \mathbb{R}^{n}$ and $b \in \mathbb{R}^{m}$. We assumes the solvability of problem (1). The least 2-norm formulation [10] of the dual linear program (2) is

$$
\left\{\begin{array}{l}
-\min b^{\top} v+\frac{\varepsilon}{2} v^{\top} v \\
A^{\top} v+c=0 \text { and } v \in \mathbb{R}_{+}^{m}
\end{array}\right.
$$

Because the objective function of (3) is strongly convex, its solution is unique. The necessary and sufficient Karush-Kuhn-Tucker optimality conditions for problem (3) give the existence of the multiplier $y \in \mathbb{R}^{n}$ such that

$$
\varepsilon v=(A y-b)_{+} \text {and } A^{\top} v+c=0,
$$

or equivalently

$$
v=\frac{1}{\varepsilon}(A y-b)_{+} \text {and } A^{\top}(A y-b)_{+}+\varepsilon c=0,
$$

where the plus function $x_{+}$is defined as

$$
\left(x_{+}\right)_{i}=\max \left(0, x_{i}\right), i=1, \ldots, n .
$$

If we consider now the parametric exterior penalty problem of the primal linear program (1) with the penalty parameter $\varepsilon$, given by the function $f$

$$
\left\{\begin{array}{l}
\min f(y)=\varepsilon c^{\top} y+\frac{1}{2}\left\|(A y-b)_{+}\right\|^{2} \\
y \in \mathbb{R}^{n},
\end{array}\right.
$$

the optimality conditions (4) become

$$
v=\frac{1}{\varepsilon}(A y-b)_{+}
$$

and

$$
\nabla f(y)=A^{\top}(A y-b)_{+}+\varepsilon c=0
$$

That is,

$$
v=\frac{1}{\varepsilon}(A y-b)_{+}
$$

and

$$
y=\arg \min _{y \in \mathbb{R}^{n}} f(y)=\varepsilon c^{\top} y+\frac{1}{2}\left\|(A y-b)_{+}\right\|^{2}
$$

Hence, solving the exterior penalty problem (5) for any positive parameter $\varepsilon$ provides a solution $v=\frac{1}{\varepsilon}(A y-b)_{+}$to (3). In addition, if $\left.\varepsilon \in\right] 0, \bar{\varepsilon}[$ for some positive $\bar{\varepsilon}$, we will have the following results.

Theorem 1. [10] The unique least 2-norm solution to the dual linear program (3) is given by

$$
v=\frac{1}{\varepsilon}(A y-b)_{+}
$$

where $y$ is a solution of the primal penalty problem (5) for any finite value of the penalty parameter $\varepsilon \in] 0, \bar{\varepsilon}[$, for some positive $\bar{\varepsilon}$.

Solving the problem 5 gives a solution $y$. We can then, compute an exact least 2-norm solution $v$ to the dual linear program (2) from (6), and if we make the assumption that the submatrix $A_{S}$ has linearly independent columns, where 


$$
S=\left\{j: v_{j}>0\right\} .
$$

As a consequence of the complementarity conditions, we have

$$
A_{j} z=b_{j}, \text { for } j \in S,
$$

and assumption (7) yields an exact solution of the primal linear program (1). From the theoretical point of view, it is not easy to find $\bar{\varepsilon}$. However, if for two successive values of $\varepsilon, \varepsilon^{1}>\varepsilon^{2}$, the corresponding solutions of problem (3) $v^{1}$ and $v^{2}$, are equal, then under certain assumptions [8], $v=v^{1}=v^{2}$, is the solution of problem (2). Computationally this can be treated effectively.

\section{Linear Programming BFGS Algorithm}

If we dispose of a fast method for solving the problem (5), the scheme proposed in section 2, can be very successful in solving large-scale linear programming.

\subsection{Linear Programming Newton Algorithm}

In [9], the author proposed a modified Newton method with Armijo step size for solving the problem (5), and since the gradient of the objective function of problem (5) is not differentiable, he used the generalized Hessian [6], which is the $n \times n$ symmetric positive-semidefinite matrix

$$
\partial^{2} f(x)=A^{\top} \operatorname{diag}(A x-b)_{*} A,
$$

where $\operatorname{diag}(A x-b)_{*}$ denotes an $m \times m$ diagonal matrix with diagonal elements $\operatorname{diag}\left(A_{i} x-b_{i}\right)_{*}$, $i=1, \ldots, m$, while $x_{*}$ denotes the subgradient of $x_{*}$ which is the step function defined as

$$
\begin{gathered}
\left(x_{*}\right)_{i}=1, \text { if } x_{i}>0 \\
\left(x_{*}\right)_{i}=0, \text { if } x_{i}<0 \\
\left(x_{*}\right)_{i} \in[0,1] \text { if } x_{i}=0 i=1, \ldots, n .
\end{gathered} .
$$

The Linear programming primal algorithm lpnewt, given in [9] was faster than CPLEX 6.5 [2] on a class of synthetically generated sparse linear programs with as many as two million constraints such that $m \gg n$. However, the method is relatively slow if $n$ approach $m$, and often gives a solution of bad quality especially when the matrix $A$ is dense. In fact, lpnewt utilizes the generalized Hessian instead of the Hessian Matrix $\nabla^{2} f$. For a quick computation of the matrix (9) at each iteration, the author in [9] was constrained to take a fixed value for $\left(x_{*}\right)_{i}$ in the interval $[0,1]$, when $x_{i}=0$, This necessarily affects the quality of the descent direction. Motivated by the fact that the BFGS method combines good convergence and simple updates for smooth unconstrained minimization problems. We present an algorithm to solve the linear program (1) based on the BFGS method to solve the problem (5).

\subsection{Linear Programming BFGS Algorithm (LPBFGS)}

We, first, state the $L P B F G S$ algorithm and then we guarantee global convergence.

\subsubsection{BFGS update formula}

The New iterate of the BFGS method applied to the the parametric exterior penalty problem of the primal linear program (5) is

$$
y_{k+1}=y_{k}+\alpha_{k} d_{k}
$$

where the Quasi-Newton search direction $d_{k}$ is

$$
d_{k}=-H_{k}\left(\nabla f\left(y_{k}\right)\right),
$$

and $H_{k}$ the approximation of the inverse Hessian matrix is updated at every step by means of Quasi-Newton-BFGS update formula given by

$$
\begin{gathered}
H_{k+1}=\left(I-\rho_{k} \Delta y_{k} \Delta f_{k}^{\top}\right) \times \\
H_{k}\left(I-\rho_{k} \Delta f_{k} \Delta y_{k}^{\top}\right)+\left(\rho_{k} \Delta y_{k} \Delta y_{k}^{\top}\right)
\end{gathered}
$$

with

$$
\Delta y_{k}=y_{k+1}-y_{k},
$$

$$
\Delta f_{k}=\nabla f\left(y_{k}\right)-\nabla f\left(y_{k}\right)
$$

and 


$$
\rho_{k}=\frac{1}{\Delta f_{k} \Delta y_{k}^{\top}} .
$$

The step length $\alpha_{k}$ is chosen to satisfy the strongly Wolfe conditions [13]:

$$
f\left(y_{k}+\alpha_{k} d_{k}\right) \leq f\left(y_{k}\right)+c_{1} \alpha_{k} \nabla f\left(y_{k}\right)^{\top} d_{k},
$$

and

$$
\left|\nabla f\left(y_{k}+\alpha_{k} d_{k}\right)^{\top} d_{k}\right| \leq c_{2}\left|\nabla f\left(y_{k}\right)^{\top} d_{k}\right|,
$$

where the constants $c_{1}$ and $c_{2}$ are chosen such that:

\begin{tabular}{|c|c|}
\hline Algo & m: Linear Programming BFGS (LPBFGS) \\
\hline 1: & $\begin{array}{l}\text { Initialize: Set the penalty parameter } \varepsilon=10^{-3} \text {, the } \\
\text { tolerance } t o l=10^{-12} \text {, the } \\
\text { step size } \alpha_{0}=1 \text { and iteration limit } k_{\max } . \\
\text { Star with any } y^{(0)} \in \mathbb{R}^{n} \text { and } H_{0} \text { (initial } \\
\text { approximation of the inverse Hessian matrix). } \\
\text { Set the counter } k=0 \text {. }\end{array}$ \\
\hline 2: & While $\left(k<k_{\max }\right.$ and $\left\|\nabla f\left(y_{k}\right)\right\|>$ tol $)$. \\
\hline 3: & Compute search direction $\quad d_{k}=-H_{k}\left(\nabla f\left(y_{k}\right)\right)$. \\
\hline 4: & Compute $\alpha_{k}$ to satisfy the Wolfe conditions and (12). \\
\hline 5: & Set $y_{k+1}=y_{k}+\alpha_{k} d_{k}$. \\
\hline 6: & Compute $H_{k+1}$ by relation (11) \\
\hline 7: & $k \leftarrow k+1$ \\
\hline 8: & End while. \\
\hline 9: & $\begin{array}{l}\text { Compute the least 2-norm dual solution } \\
v^{*}=\frac{1}{\varepsilon}\left(A y_{k}-b\right)_{+}\end{array}$ \\
\hline 10: & Select the set indice $S=\left\{j: v_{j}^{*}>0\right\}$ \\
\hline 11: & Solve the linear system 8 to obtain the solution $z^{*}$ \\
\hline End. & \\
\hline
\end{tabular}
$0<c_{1}<c_{2}<1$ (typically for this algorithm; $10^{-3}$ and 0.6 respectively).

3.2.2 Bounded Spectrum of the Inverse Hessian Approximation

When the objective function is twice continuously differentiable, a classic assumption for the convergence of the BFGS method [13], from any starting point $y^{0}$ and any initial Hessian approximation $H_{0}$, is that, the Hessien matrix is positive definite on the level set,

$$
\operatorname{Lev}_{f}\left(f\left(y^{0}\right)\right)=\left\{y \in \mathbb{R}^{n}: f(y) \leq f\left(y^{0}\right)\right\} .
$$

This assumption implies that there exist positive constants $m$ and $M$ such that

$$
m\|x\|^{2} \leq y^{T} \nabla^{2} f(y)^{-1} y \leq M\|x\|^{2}
$$

for all $x \in \mathbb{R}^{n}$ and $y \in \operatorname{Lev}_{f}\left(f\left(y^{0}\right)\right)$. Form the analysis given in [13], we can translate the bounds on the spectrum of $H_{k}$ into conditions that only involve $\Delta y_{k}$ and $\Delta f_{k}$. We get then

$$
m \leq m_{k}=\frac{\Delta f_{k}^{\top} \Delta y_{k}}{\Delta y_{k}^{\top} \Delta y_{k}} \leq \frac{\Delta f_{k}^{\top} \Delta f_{k}}{\Delta f_{k}^{\top} \Delta y_{k}}=M_{k} \leq M .
$$

It is Impossible to guarantee (14) for our problem (5). However, We do not tolerate for $L P B F G S$ Algorithm, having a too small $m_{k}$ and too large $M_{k}$. In the first experimental tests we took this into account. But we observed that this condition has never been invoked for the values $10^{-3}$ and $10^{8}$ of $\mathrm{m}$ and $M$ respectively, we therefore omitted.

Theorem 2. Let the sequence $\left(y_{k}\right)_{k \in \mathbb{N}}$ generated by Algorithm.

(i) Then either $\left(y_{k}\right)_{k \in \mathbb{N}}$ terminates at a solution $y^{(k)}$ or every accumulation point $y^{*}$ of $\left(y_{k}\right)_{k \in \mathbb{N}}$ solve problem (5).

(ii) The corresponding $v^{*}=\frac{1}{\varepsilon}\left(A y^{*}-b\right)_{+}$is the exact least 2-norm solution to the dual linear program (2), for some sufficiently small $\varepsilon$.

(iii) If the assumption 7 is checked for

$$
S=\left\{j: v_{j}^{*}>0\right\},
$$

an exact solution $z^{*}$ to the primal linear program (1) is obtained by step 12 of the algorithm.

Proof. We prove only (i), (ii) and (iii) follows immediately from ([9], Theorem 3.1). The solvability of problem (1) involves that of (5). Let the sequence $\left(y_{k}\right)_{k \in \mathbb{N}}$ generated by algorithm 1 , and 
suppose that $\nabla f\left(y_{k}\right) \neq 0$, there exists a subsequence $\left(y_{k_{n}}\right)_{n \in \mathbb{N}}$ that converges to the accumulation point $y^{*}$ of $\left(y_{k}\right)_{k \in \mathbb{N}}$. By the first Wolfe conditions (12) and the Quasi-Newton direction (10), we get $f\left(y_{k_{n}}\right)-f\left(y_{k_{n}+1}\right) \geq c_{1} \alpha_{k} \nabla f\left(y_{k_{n}}\right)^{\top} H_{k}\left(\nabla f\left(y_{k_{n}}\right)\right) \geq 0$.

Then, the sequence $\left(f\left(y^{\left(k_{n}\right)}\right)\right)_{n \in \mathbb{N}}$ is nonincreasing and converges to the point $f\left(y^{*}\right)$. Since the gradient of $f \quad$ is Lipschitz continuous with constant $\left\|A^{\top}\right\|\|A\|$, we have

$$
\left\|\nabla f\left(y_{k_{n}}\right)-\nabla f\left(y^{*}\right)\right\| \leq\left\|A^{\top}\right\|\|A\|\left\|y_{k_{n}}-y^{*}\right\|,
$$

we deduce that $\left(\nabla f\left(y_{k_{n}}\right)\right)_{n \in N}$ converges to $\nabla f\left(y^{*}\right)$. By letting $n \rightarrow \infty$ in (16) and the fact that $H_{k}$ always check (15) we get

$$
\nabla f\left(y^{*}\right)=0 .
$$

Remark 3. In the proof of the last theorem, we have used only the first strong Wolfe condition called the Armijo stepsize or also, the sufficient decrease condition. However, the second strong Wolfe condition called the curvature condition, is sufficient to guarantee the so called secant equation formula, i.e. $H_{k+1} \Delta f_{k}=\Delta y_{k}$, which must satisfy the updated approximation $H_{k+1}$. For more comprehension on the BFGS method, we refer the reader to the classical reference [13].

\section{Numerical Tests}

The objective of our numerical experiments is to show that the lpbfgs method is more effective than lpnewt when $m \simeq n$. Recall that when $m>n$ lpnewt was better than CPLEX 6.5 [2] that uses an efficient interior-point method. Firstly, we have implemented the lpnewt procedure whose
MATLAB [12] code is given explicitly in ( [9], page 13). Secondly we have implemented Algorithm 1 in the same language. Both algorithms was tested on the same very large synthetically generated test problems and on the same Intel (R) Core (TM)2 Duo CPU T5870@2.00 GHz with 3 Go of RAM.The test problem generator, called lpgen by the author in [9], generates a random constraint matrix $A$ for given $(m, n)$, and density $d$. The elements of $A$ are uniformly distributed between -50 and +50 . A primal random solution $x$, with elements in $[-10,10]$, and a dual solution $u$, with elements in $[0,10]$. These solutions are then used to generate an objective function cost vector $c$ and a right-hand side vector $b$ for the linear program (1). For the lpnewt the parameter values $\delta, \varepsilon$ and tolerance tol are taken as in [9] $\left(10^{-3}, 10^{-4}\right.$, and $10^{-12}$ respectively). For both procedures, the iteration limit $k_{\max }$ is taken equal to 100 , and the starting point $y^{(0)}$ is typically equal to $\left(\bar{A}^{\top} \bar{A}+\varepsilon I\right)^{-1} \bar{A}^{\top} \bar{b}$, where $\bar{A}$ is an arbitrary $n \times n$ subset of $A$, and $\bar{b}$ is the corresponding $n \times 1$ subset of $b$.

In Table 1, we give the comparison between the two methods, when, depending on CPU time (columns 4 and 6) and quality solution (columns 3 and 5); where $Z_{*}$ denotes the exact solution, $Z_{\text {lpbfgs }}$ and $Z_{\text {lpnewt }}$ are the solutions given by $L P B F G S$ and lpnewt algorithms respectively and the norm \|\| is the Euclidean norm. Column 2 represent the density $d$ of the matrix system $A$. The best values are in bold. With the exception of the last example, the quality of the solution given by the $L P B F G S$ method is much higher than that given by the method lpnewt . However, In table 2 we give a comparison when $m=n$. We had to be limited to $n=10^{3}$, because beyond lpgen is out of memory, and as we see in Table 2, the difference is huge when $m \simeq n$. 
Table 1 Comparison of LPBFGS and lpnewt when $m>n$.

$\begin{array}{cccccc}(m, n) & d & \left\|Z_{*}-Z_{\text {lpbfgs }}\right\| & \text { Time }(\text { lpbfgs }) & \left\|Z_{*}-Z_{\text {lpnewt }}\right\| & \text { Time(lpnewt }) \\ 10^{3} \times 10^{2} & 0.1 & 0.3548 & 0.7824 & 165.6835 & 0.0585 \\ 10^{3} \times 10^{2} & 1 & 1.0822 \mathbf{e}-013 & 1.3813 & 84.1570 & 0.1264 \\ 10^{4} \times 10^{2} & 0.1 & 0.5462 & 2.4017 & 31.0228 & 0.1751 \\ 10^{4} \times 10^{2} & 1 & 0.0988 & 6.8536 & 37.3349 & 0.8189 \\ 10^{5} \times 10^{2} & 0.1 & 0.2768 & 16.6980 & 243.6130 & 3.7684 \\ 10^{5} \times 10^{2} & 1 & 5.2354 \mathbf{e}-014 & 59.3134 & 36.2556 & 16.4920 \\ 10^{6} \times 10^{2} & 0.05 & 0.3070 & 607.0350 & 1.4492 \mathbf{e}-014 & 195.9422\end{array}$

Table 2 Comparison of LPBFGS and lpnewt when $m=n$.

$\begin{array}{cccccc}(m, n) & d & \left\|Z_{*}-Z_{\text {lpbfgs }}\right\| & \text { Time }(\text { lpbfgs }) & \left\|Z_{*}-Z_{\text {lpnewt }}\right\| & \text { Time }(\text { lpnewt }) \\ 10^{3} \times 900 & 0.1 & 9.0547 \mathbf{e}-014 & 9.4193 \mathbf{e}+000 & 5.4984 e+002 & 2.2633 e+001 \\ 10^{3} \times 900 & 1 & 4.7073 \mathbf{e}-014 & 1.7154 \mathbf{e}+001 & 2.3862 e+001 & 1.7068 e+002 \\ 10^{3} \times 950 & 0.1 & 4.4578 \mathbf{e}-014 & 1.0506 \mathbf{e}+001 & 1.6543 e+002 & 2.6286 e+001 \\ 10^{3} \times 950 & 1 & 4.9370 \mathbf{e}-014 & 1.7268 \mathbf{e}+001 & 6.6964 e+001 & 2.0234 e+002 \\ 10^{3} \times 990 & 0.1 & 3.8192 \mathbf{e}-014 & 9.6128 \mathbf{e}+000 & 9.8499 e+001 & 2.2491 e+001 \\ 10^{3} \times 990 & 1 & 4.2633 \mathbf{e}-014 & 1.5928 \mathbf{e}+001 & 1.0844 e+002 & 1.6980 e+002\end{array}$

\section{Conclusion}

It is known [13] that the $B F G S$ formula has very effective self-correcting properties. When the rounding errors that occur infinite-precision implementation, or when the matrix $H_{k}$ incorrectly estimates the curvature in the objective function, then the Hessian approximation (11) will tend to correct itself within a few steps. This explains the difference in quality between the two methods. Having obtained very satisfactory results, we was merely limited to the BFGS method with strongly Wolfe linear search. However, a method like the Limited-memory BFGS quasi-Newton methods which is useful for solving large problems can also give better results.

\section{References}

[1] R. H. Byrd and J. Nocedal, A tool for the analysis of quasi-Newton methods with application to unconstrained minimization, SIAM J. Numer. Anal., 26 (1989), pp. 727-739.
[2] Cplex Optimization, Incline Village, Nevada, Using the CPLEX(TM) Linear Optimizer and CPLEX(TM) Mixed Integer Optimizer (Version 2.0), 1992.

[3] Glenn Fung, O.L. Mangasarian, Breast tumor susceptibility to chemotherapy via support vector machines. CMS 3:103-112 (2006).

[4] Glenn Fung, O.L. Mangasarian, A Feature Selection Newton Method for Support Vector Machine Classification. Computational Optimization and Applications, 28, 185-202 (2004).

[5] A. I. Golikov and Y. G. Evtushenko, Search for Normal Solutions in Linear Programming, Computational Mathematics and Mathematical Physics, Vol. 14, pp. 1694-1714, 2000.

[6] J. B. Hiriart-urruty, J. J. Strodiot, and V. H. Nguyen, Generalized Hessian Matrix and Second-Order Optimality Conditions for Problems with CL1 Data, Applied Mathematics and Optimization, Vol. 11, pp. 43-56, 1984.

[7] A. S. Lewis and M. L. Overton. Nonsmooth optimization via BFGS. Technical report, Optimization Online, 2008a. URL. http://www.optimization-online.org/DB_FILE/ 2008/12/2172. pdf. Submitted to SIAM J.Optimization.

[8] LUCIDI, S., A New Result in the Theory and Computation of the Least-Norm Solution of a Linear 
Program, Journal of Optimization Theory and Applications, Vol. 55, pp. 103-117, 1987.

[9] O. L. Mangasarian, A Newton Method for Linear Programming,Journal of Optimization Theory and Applications: vol. 121, No. 1, pp. 1-18, 2004.

[10] O. L. Mangasarian, and MEYER, R. R., Nonlinear Perturbation of Linear Programs,SIAM Journal on Control and Optimization, Vol. 17, pp. 745-752, 1979.

[11] O. L. Mangasarian, Normal Solutions of Linear Programs, Mathematical Programming Study, Vol. 22, pp. 206-216, 1984.

[12] MATLAB User's guide. http://www.mathworks.com
[13] J. Nocedal and S. J. Wright. Numerical Optimization. Springer Series in Operations Research. Springer, 1999.

[14] C. Roos, T. Terlaky and J. P. Vial, Interior Point Methods For Linear Optimization. Springer Science+Business Media, Inc (2005).

[15] A.N. Tikhonov and V.Y. Arsenin: Solutions of ill-posed problems. Halsted Press, Wiley, New York, NY, 1977.

[16] J. Yu, S.V.N. Vishwanathan, S. Gunter, N. N. Schraudolph, A Quasi-Newton Approach to Nonsmooth Convex Optimization Problems in MachineLearning. Journal of Machine Learning Research 11: 1145-1200 (2010). 